\title{
Konzentriertes Mahlzeiteninsulin
}

\section{Die Vorteile kommen im Alltag an}

_ Immer mehr adipöse Menschen benötigen prandiales Insulin, um ihr Risiko für Folgekomplikationen in Schach zu halten. Einer Untersuchung in Europa zufolge brauchten im Jahr 2012 mehr als $70 \%$ der Betroffenen über $20 \mathrm{E} / \mathrm{d}$, wie Dr. Jens Kröger, Hamburg, berichtete. Auch er beobachte in der Praxis eine steigende Tendenz bei den Insulindosen. Für immer mehr Patienten würde dadurch mit niedrig konzentrierten Insulinen eine Mahlzeitengabe von jeweils zwei Injektionen nötig (Splitting).

\section{Mehr Patienten- und Arztkomfort}

Daher wurde das prandiale Insulinanalogon Lispro in einer höher konzentrierten Form mit $200 \mathrm{E} / \mathrm{ml}$ (Liprolog $\left.{ }^{\circledR} 200\right)$ in einem angepassten KwikPen entwickelt.
Eine Online-Umfrage unter 60 Diabetologen und 150 Hausärzten offenbarte, dass damit ins Schwarze getroffen wird: Die Befragten erhoffen sich von dem neuen Insulin u. a. weniger Splitting, weniger Gewebereaktionen, eine längere Reichweite des Pens mit weniger Einbestellungen, weniger Müll und geringere Zuzahlungen.

Die Vorteile kämen bereits bei der Ersteinstellung zum Tragen, so Kröger. Die Umstellung von Lispro $100 \mathrm{E} / \mathrm{ml}$ sei einfach, da der spezielle KwikPen für Lispro $200 \mathrm{E} / \mathrm{ml}$ keine veränderte Handhabung verlangt.

Wichtig zu beachten ist, dass das hoch konzentrierte Insulin, das nur im Fertigpen verfügbar ist, trotz Bioäquivalenz zu Lispro $100 \mathrm{E} / \mathrm{ml}$ nicht für den i.v.-Einsatz,

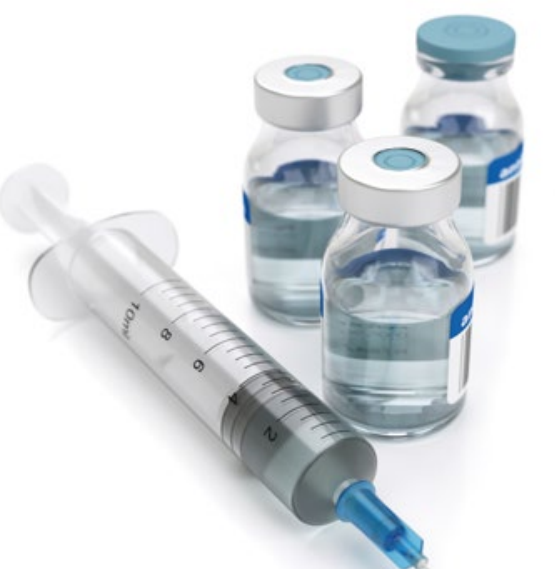

Für viele Diabetiker reicht periprandial eine Insulindosis nicht aus.

für Insulinpumpen und für Kinder zugelassen ist. Außerdem ist der Pen aufgrund der Haltbarkeit von 28 Tagen nach Anbruch nur für Patienten empfohlen, die $>$ $20 \mathrm{E} / \mathrm{d}$ prandiales Insulin brauchen.

\section{Sarah Louise Pampel}

- Presse-Round-Table „Doppelt konzentriert, weniger injiziert: Liprolog ${ }^{\circledR} 200$ in der Praxis angekommen, von Patienten angenommen", Herbsttagung der Deutschen Diabetes-Gesellschaft; Düsseldorf, November 2015 (Veranstalter: Berlin-Chemie)

\section{HIV-Therapie}

\section{Eine Tablette - drei Wirkstoffe}

_ Gemäß Leitlinien erfolgt die antiretrovirale Behandlung bei einer Infektion mit dem Humanen ImmundefizienzVirus (HIV) mit einer Kombination von drei Wirkstoffen, die mit nur einer Tablette pro Tag eingenommen werden können. Die Erfolgsraten, gemessen als dauerhafte Reduktion der HIV-RNA unter 50 Kopien/ml, liegen bei über $90 \%$. Nur bei wenigen Patienten spricht die Therapie nicht an, selten kommt es zu Resistenzen. Frühzeitiger Therapiebeginn, unabhängig von der T-Helferzellen-Zahl, verbessert die Prognose nochmals, wie die sogenannte START-Studie zeigen konnte.

\section{Verbesserung des zentralen Therapiebausteins}

Ein besonders wichtiger Baustein der antiretroviralen Kombinationstherapie ist der nukleosidale Reverse-Transkriptase-Inhibitor (NRTI) Tenofovir, den die Firma Gilead in Monotherapie (Viread $\left.{ }^{\star}\right)$ sowie in mehreren Kombinationstherapien (Truvada ${ }^{\oplus}$, Atripla ${ }^{\oplus}$, Eviplera ${ }^{\circledR}$ und Stribild ${ }^{\circledR}$ anbietet.

Tenofovir wird als Prodrug (Tenofovirdisoproxil-Fumarat) eingenommen. Nur so gelangt der Wirkstoff in die Zelle. Allerdings muss er mit $245 \mathrm{mg} / \mathrm{d}$ relativ hoch dosiert werden, um ausreichende Wirkspiegel in den Zielzellen zu erreichen. Viel Wirkstoff verbleibt im Plasma, was langfristig zu Nebenwirkungen führen mag.

Nun hat Gilead eine neue ProdrugForm entwickelt, Tenofovir-Alafenamid. Diese reichert sich deutlich stärker in den Zielzellen an, weil sie erst dort gespalten wird, erklärte Prof. Georg Beh- rens, Hannover. Entsprechend kann Tenofovir um den Faktor 10 niedriger dosiert werden. Die Plasma-Exposition sinkt um 91\%, was zu geringeren Nebenwirkungen (signifikant geringerer Rückgang der Knochendichte und seltenere Proteinurie) führt.

Der Hersteller wird Tenofovir-Alafenamid in allen bisherigen Kombinationen entwickeln. Den Auftakt macht die die Dreifachkombination Genvoya ${ }^{\oplus}$. Sie enthält den Integrase-Inhibitor Elvitegravir, den pharmakokinetischen Booster Cobicistat, den nukleotidalen Reverse-Transkriptase-Inhibitor (NtRTI) Emtricitabin und TenofovirAlafenamid.

\section{Dr. Dirk Einecke}

- Einführungs-Pressekonferenz Genvoya ${ }^{\oplus}$ "HIV heute: Perspektive Zukunft"; Frankfurt, Dezember 2015 (Veranstalter: Gilead) 